\title{
The new WMM2020 and IGRF-13 models, and a retrospective analysis of IGRF secular variation
}

William Brown, Ciarán Beggan, Grace Cox, Susan Macmillan

\author{
British Geological Survey, UK
}




\section{Abstract}

2020 marks the start of a new 5-year cycle and updated releases of the World Magnetic Model (WMM) and International Geomagnetic Reference Field (IGRF). These models provide a reference for the up-to-date internal geomagnetic field in 2020, and a prediction of its secular variation for the next 5 years, to 2025. While similar in some aspects, the two models have different specifications and many different users across diverse fields. They provide references to be used primarily for navigation (WMM) and geomagnetic coordinate systems (IGRF).

BGS produces the WMM in collaboration with the US' NOAA/NCEI, while the IGRF is produced by an IAGA Div. V-MOD task force, this time consisting of fifteen teams across nine nations, including BGS. Here we present a summary of the updated WMM2020, and BGS efforts to enable access to these models.

We also present a retrospective analysis of the predictive components of the candidate models for the previous IGRF epoch's secular variation. Recent epochs have seen notable geomagnetic jerks and the acceleration of the North magnetic dip pole, features not well represented by the constant SV format of models such as the IGRF. We assess the range of candidate models submitted for the previous IGRF epoch, assess the accuracy of physically derived predictions versus empirical extrapolations, and discuss the implications given the range of candidate models submitted for IGRF13 secular variation over the next five years. 


\section{Overview}

1. WMM2020

2. IGRF-13

3. BGS access to models

4. Retrospective look at IGRF-12 secular variation candidates

5. Summary

6. References 


\section{WMM2020}

- World Magnetic Model is produced jointly by BGS (UK) and NOAA/NCEI (USA)

- Commissioned by UK Defence Geographic Centre and US National Geospatial-Intelligence Agency

- Widely used for navigation by industry, government, military organisations

- Spherical harmonic degree 12 model of main field and secular variation

- Snapshot of field at 2020.0, and constant SV projection to 2025.0

- Includes error model estimating uncertainty from modelling process, exclusion of crustal and disturbance fields

- Software, maps and online calculators available from NOAA/NCEI WMM homepage:

https://www.ngdc.noaa.gov/geomag/WMM/

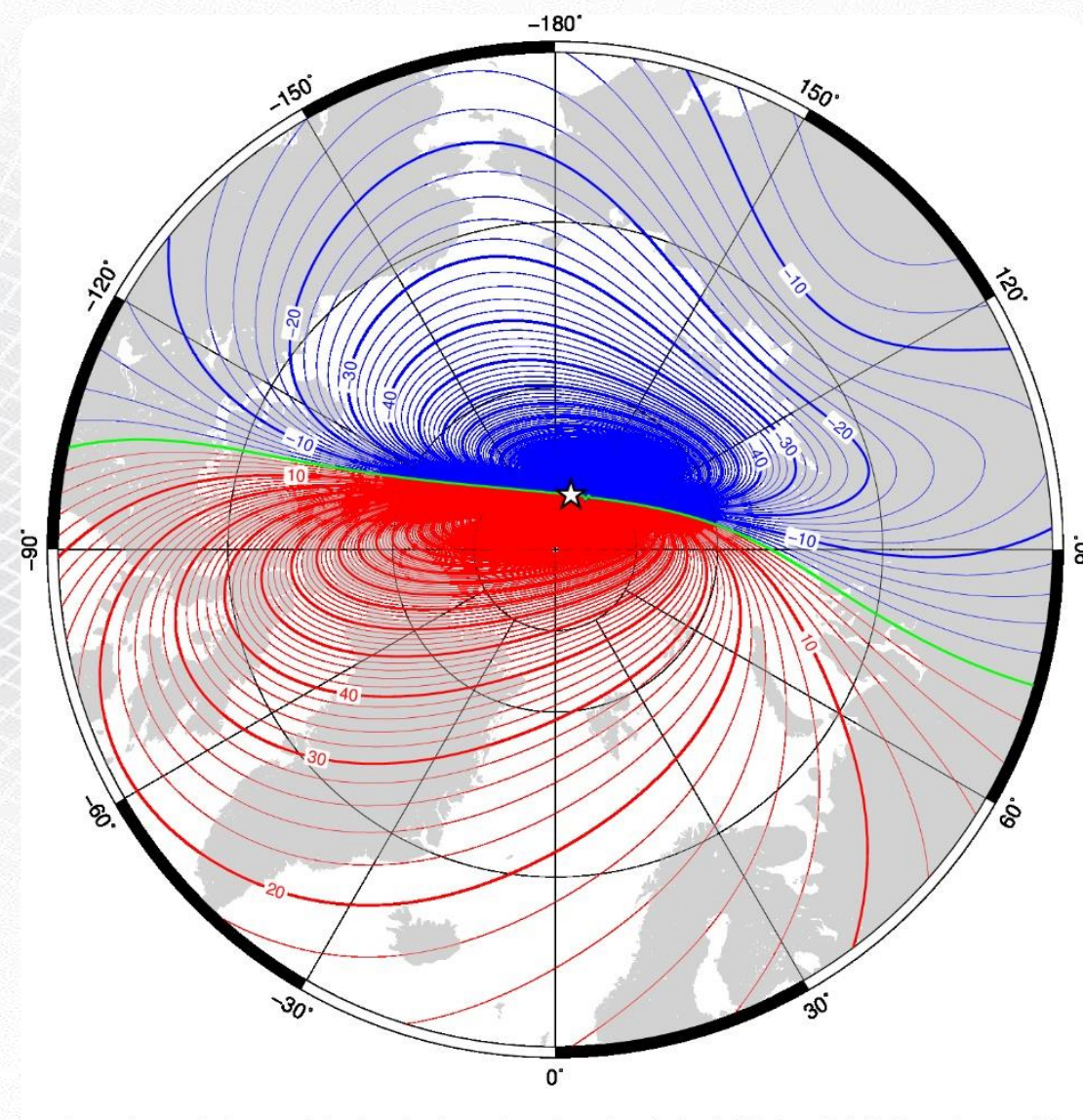

Annual rate of change of declination in region of north pole for 2020.0 to 2025.0 from the World Magnetic Model (WMM2020). Red - easterly change, blue - westerly change, green - zero change. Contour interval is $2 \%$ year, white star is location of a magnetic pole and projection is Polar Stereographic. Credit: British Geological Survey (UK Research and Innovation). 


\section{IGRF-13}

- International Geomagnetic Reference Field is produced collaboratively by IAGA Working Group V-MOD

- Contributions from 15 teams across 9 nations

- Widely used as a reference main field for geomagnetic coordinate systems

- Spherical harmonic degree 13 model of main field and degree 8 of secular variation

- Snapshot of field at 2020.0, and constant SV projection to 2025.0

- Snapshots of field each 5 years from 1900.0

- Software (C, Fortran, Python) available from IAGA V-MOD homepage:

https://www.ngdc.noaa.gov/IAGA/vmod/igrf.html

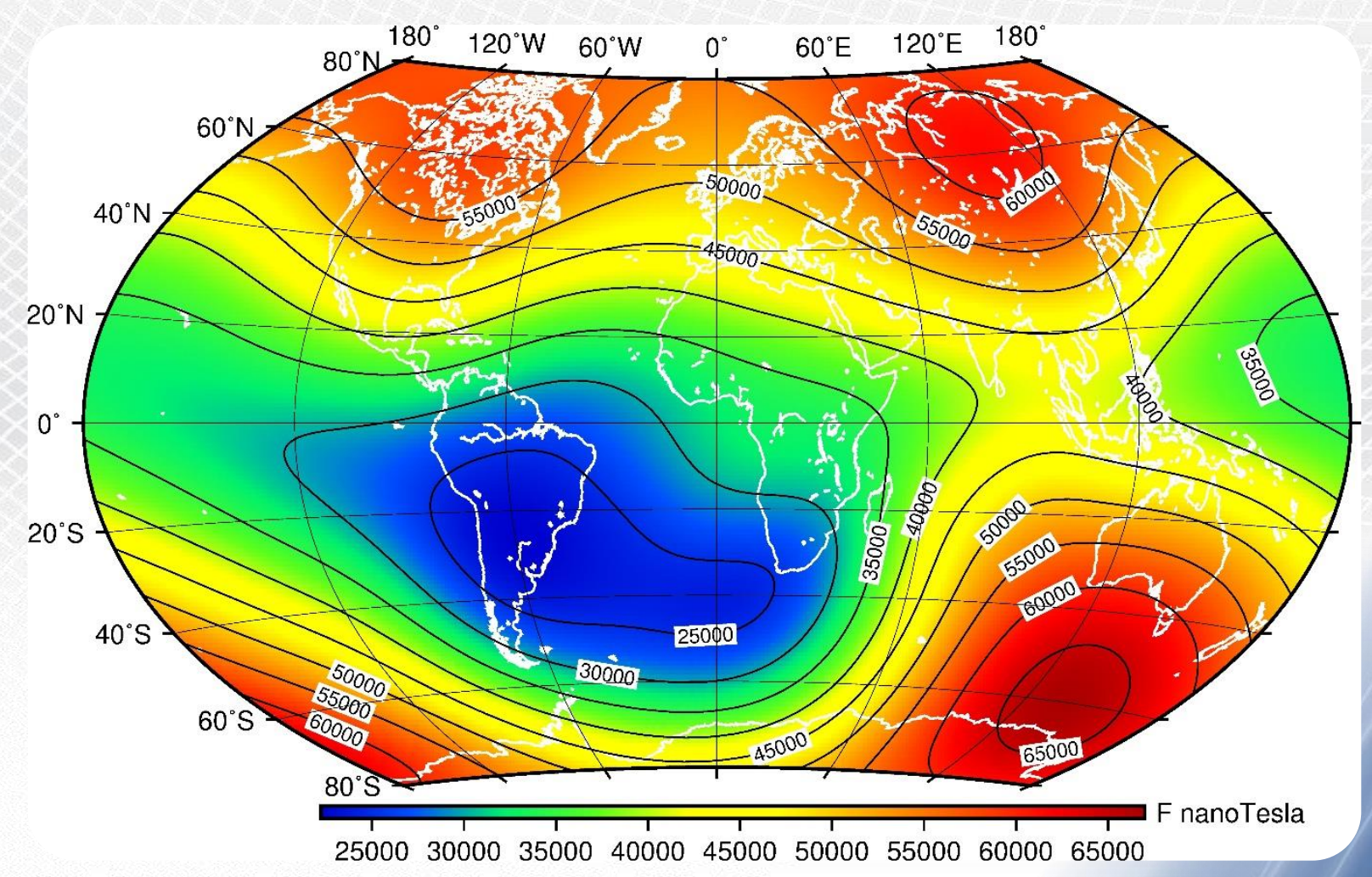




\section{BGS access to models}

- Online calculators provide access to:

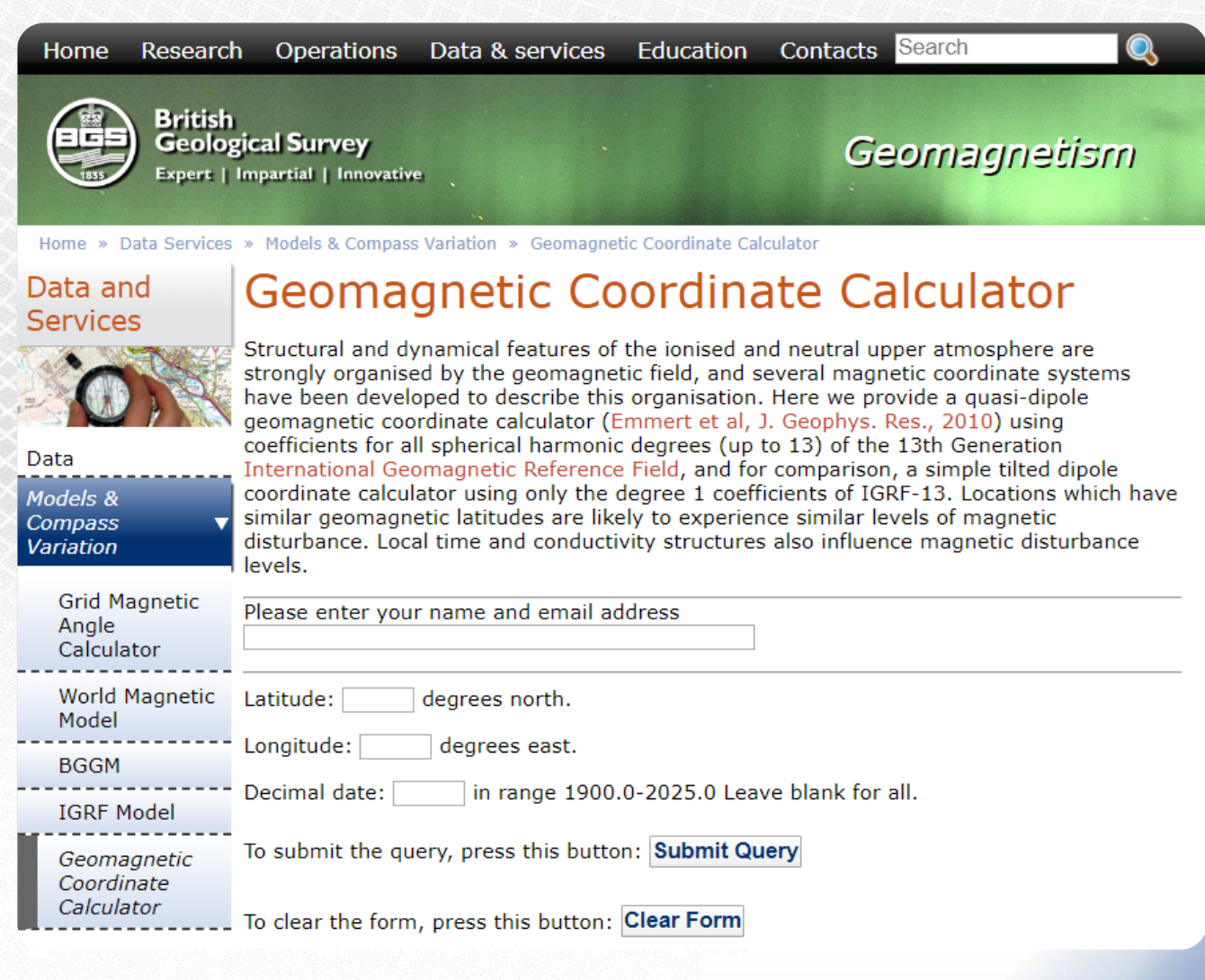

- WMM2020

- IGRF-13

- geomagnetic coordinate conversions (IGRF-13 dipole, quasi-dipole)

- Interactive or programmatic access to model values

- Maps of all field components

https://geomag.bgs.ac.uk/data service/models compass 


\section{Magnetic pole locations}

- North and South, geomagnetic and dip, pole coordinates and maps available

Locations of the north and south dip poles and geomagnetic poles. Locations (geodetic) from the $13^{\text {th }}$ Generation International Geomagnetic Reference Field

\begin{tabular}{|c|c|c|c|c|c|c|c|c|c|}
\hline \multicolumn{3}{|c|}{ North dip pole } & \multicolumn{3}{c|}{ South dip pole } & \multicolumn{2}{c|}{$\begin{array}{c}\text { North geomagnetic } \\
\text { pole }\end{array}$} & \multicolumn{2}{c|}{$\begin{array}{c}\text { South geomagnetic } \\
\text { pole }\end{array}$} \\
\hline Epoch & Latitude & Longitude & Latitude & Longitude & Latitude & Longitude & Latitude & Longitude \\
\hline 2015.0 & 86.31 & -160.47 & -64.28 & 136.60 & 80.37 & -72.61 & -80.37 & 107.39 \\
\hline 2020.0 & 86.49 & 162.76 & -64.08 & 135.86 & 80.65 & -72.68 & -80.65 & 107.32 \\
\hline 2025.0 & 85.78 & 138.09 & -63.85 & 135.06 & 80.90 & -72.64 & -80.90 & 107.36 \\
\hline
\end{tabular}

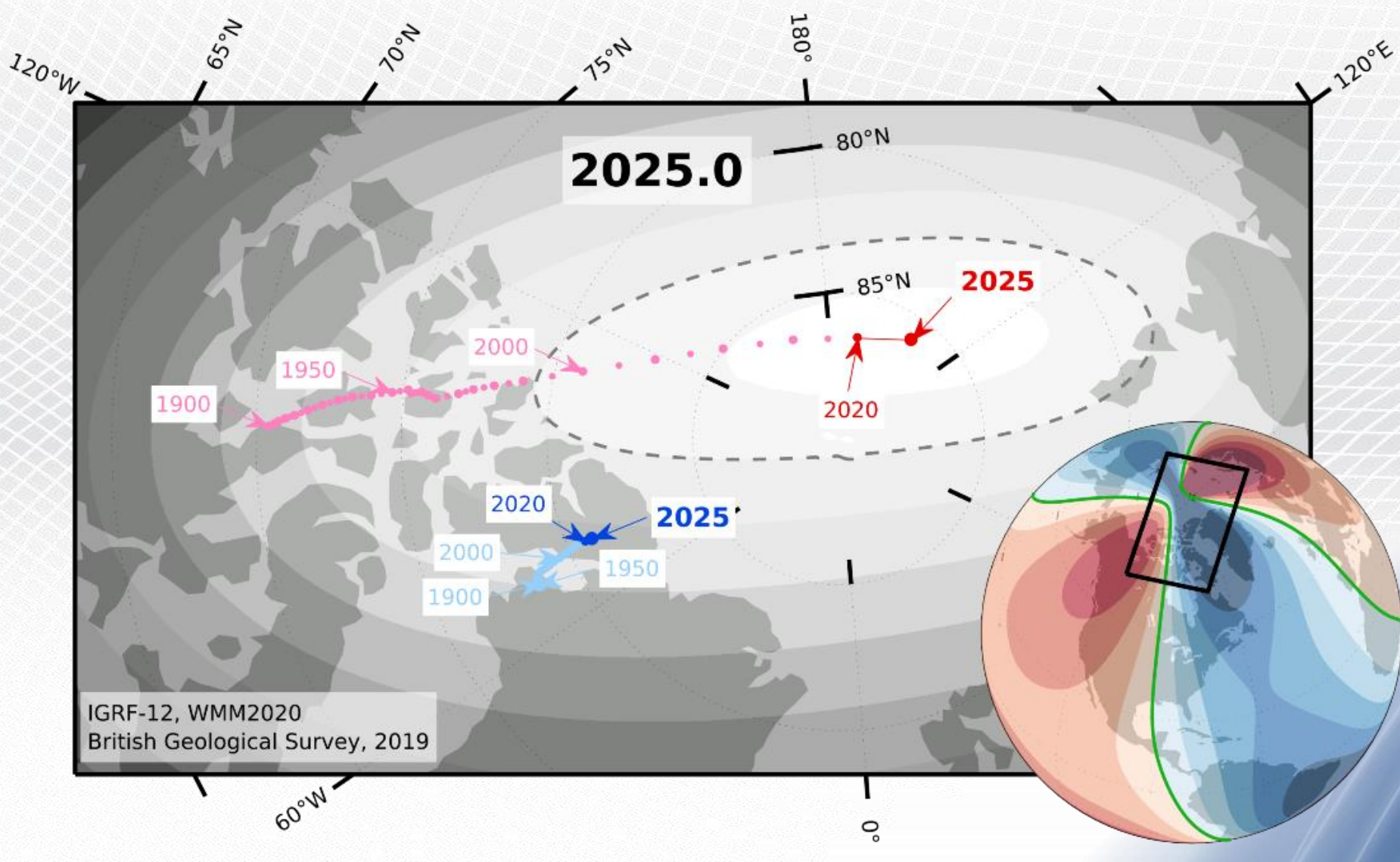

https://geomag.bgs.ac.uk/education/poles.html 


\section{IGRF-12 SV candidates}

- The previous $12^{\text {th }}$ generation of IGRF (2015-2020) provides a useful review of how a linear SV model copes with rapid and non-linear SV

- 9 candidates submitted, 4 used empirical extrapolations, 5 used physical forecasts (see Thébault, et al., 2015)

- Is there a clear distinction in the performance of the empirical or physical SV predictions? Or did any one model out-perform the others?

- Compare IGRF-12 candidates to IGRF-13 SV, produced retrospectively 


\section{IGRF-12 SV candidates}

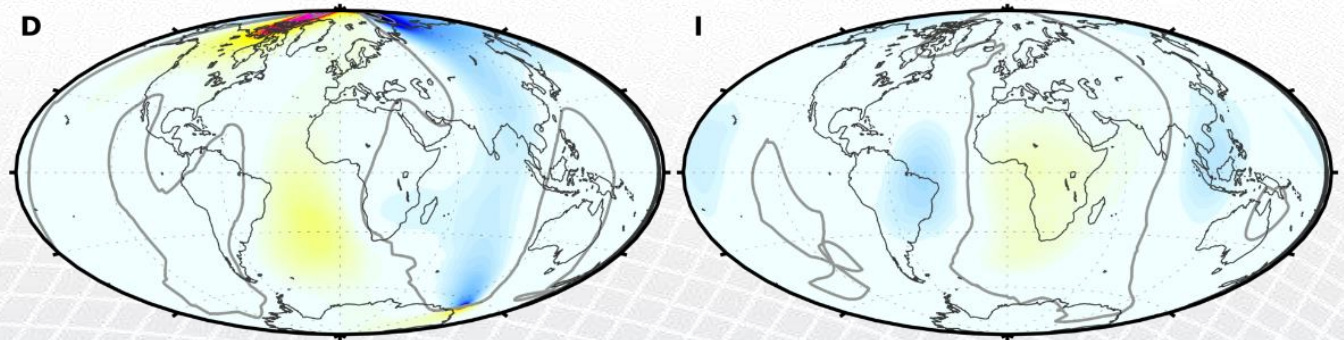

- The median of field differences between all SV candidates and IGRF-13 SV for 2015-2020 show anomalies are consistent across candidates
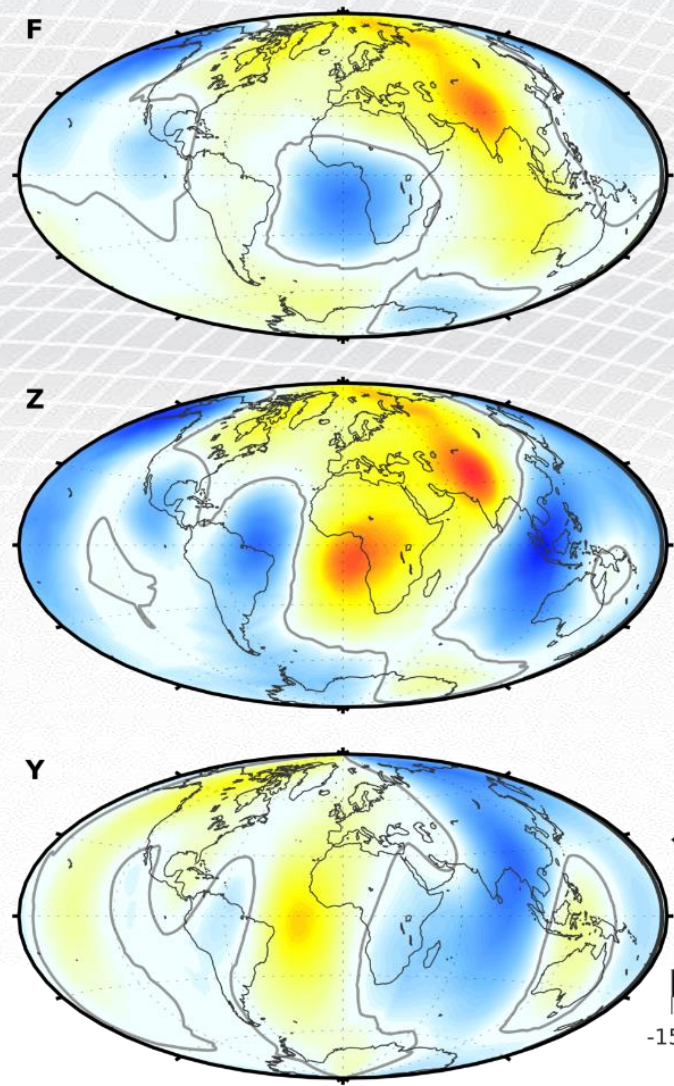

(c) UKRI All rights reserved
H

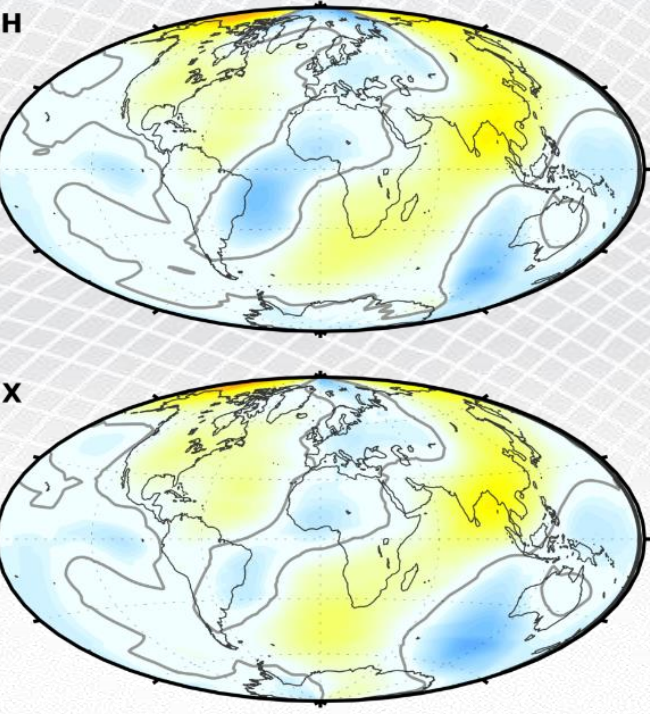

Median IGRF-12

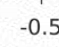

1

0.5

Degrees.yr

$-150$

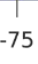

TT.yr

- Highlights the regions of greatest SV, particularly Z, and shift of North dip pole (see D, X, H above Canada)

- SV magnitude underestimated by IGRF-12 candidates due to acceleration of field in these regions

- $\quad Z$ corresponds to the regions seeing jerk activity (e.g. Torta et al., 2015), lacking from all candidates

- Differences show higher SH degrees needed to fit peak amplitudes of SV

- South Atlantic Anomaly decay also accelerating 


\section{IGRF-12 SV candidates}

- The spatial map of erroneous SV predictions is largely static in time, but amplitude varies

- The features not captured by IGRF-12 SV candidates build over several years, are not short lived

- Differences between IGRF-13 and a recent BGS model, using temporal B-splines to capture SA, provides a proxy for the amount of non-linear SV occurring

- IGRF type predictions increasingly diverge, in a linear sense for the SV predicted to SH degree 8, and increasingly so when the missing smaller scales to degree 13 are also considered

- Degrees 9-13 contribute a base RMS error of $140 \mathrm{nT}$ to forecasts

- No clear distinction between forecast methods

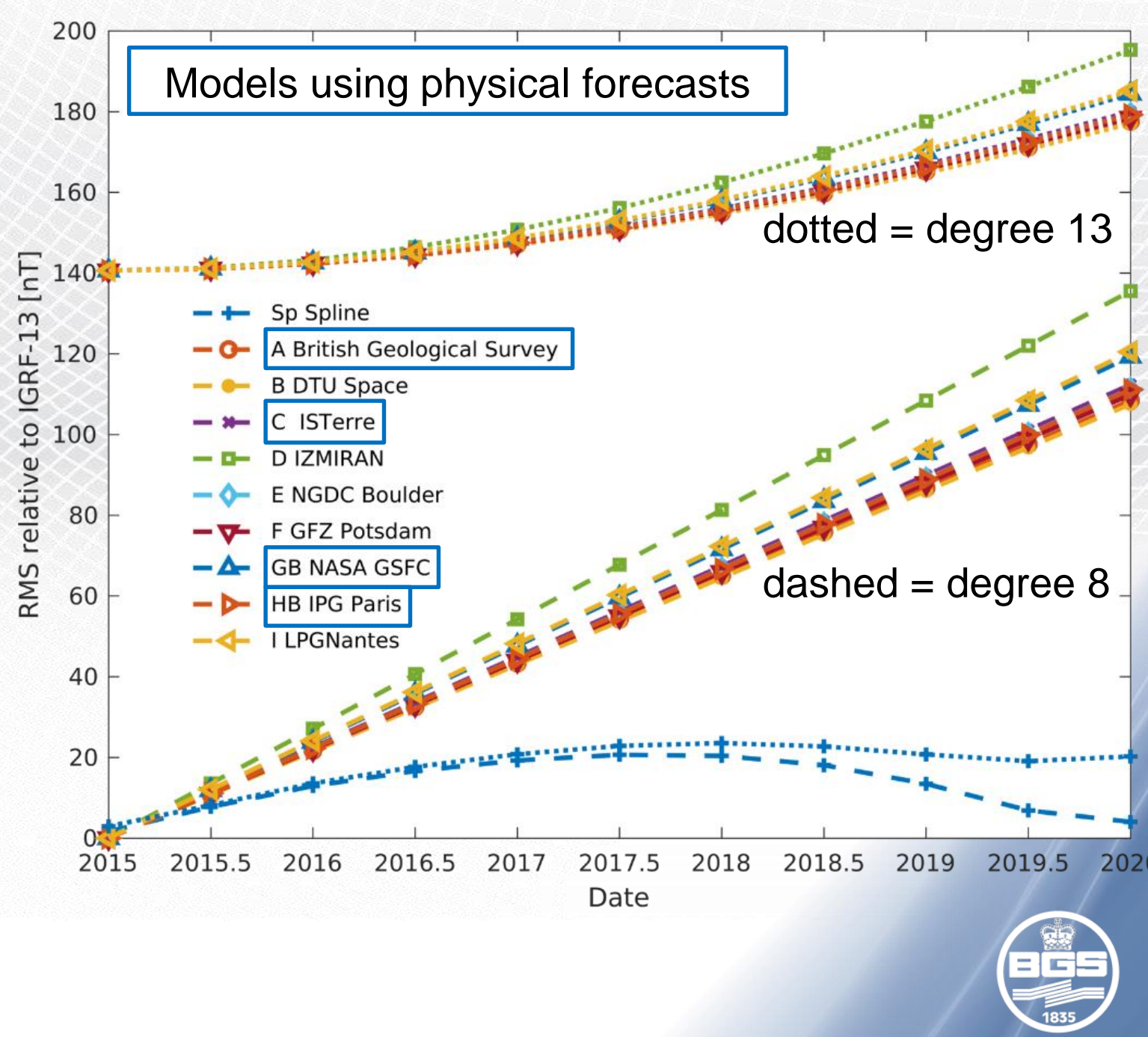




\section{IGRF-12 SV candidates}

- Best performing models use core flow advection and empirical linear extrapolation of Gauss coefficients

- $\quad$ All models are comparable with respect to errors in regions of SA, but relatively worst performing models also contain unrelated anomalies in other areas - generally, all candidates perform comparably to each other

- Suggests that use of simple extrapolations is not unfounded given the parameters of the IGRF design, but also that physical forecast of linear SV are plausible on these temporal and spatial scales

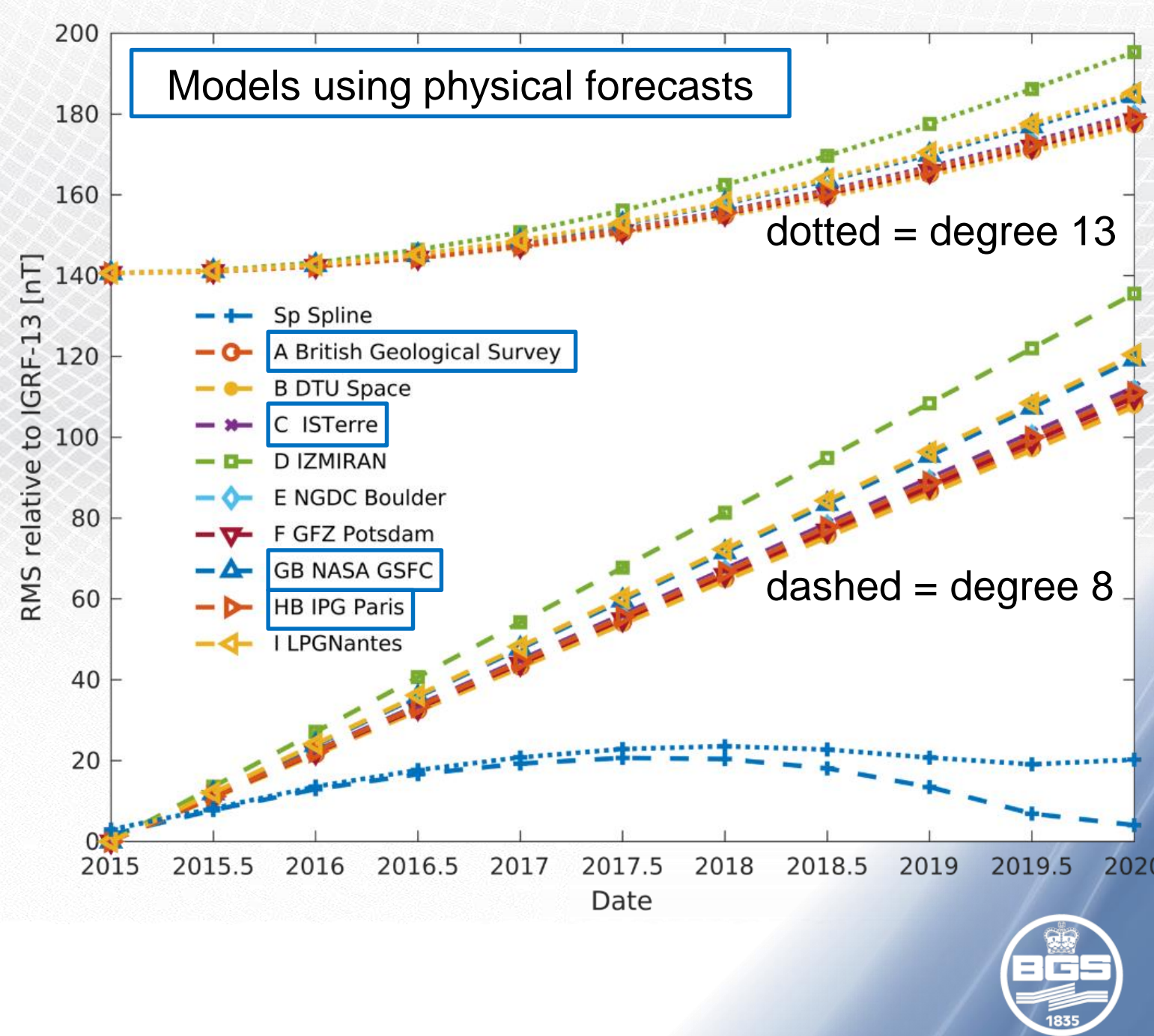




\section{IGRF-13 SV candidates}

- Timeliness of data used to build model likely has as significant an impact on SV forecasts as the method used, and is evident in IGRF-13 SV candidates

- A few weeks or months additional data generally doesn't greatly impact spatial patterns of SV, but alters amplitudes (e.g. in Z) where acceleration has occurred

Data to July 2019

BGS model @ 2020.0 - IGRF-13 SV
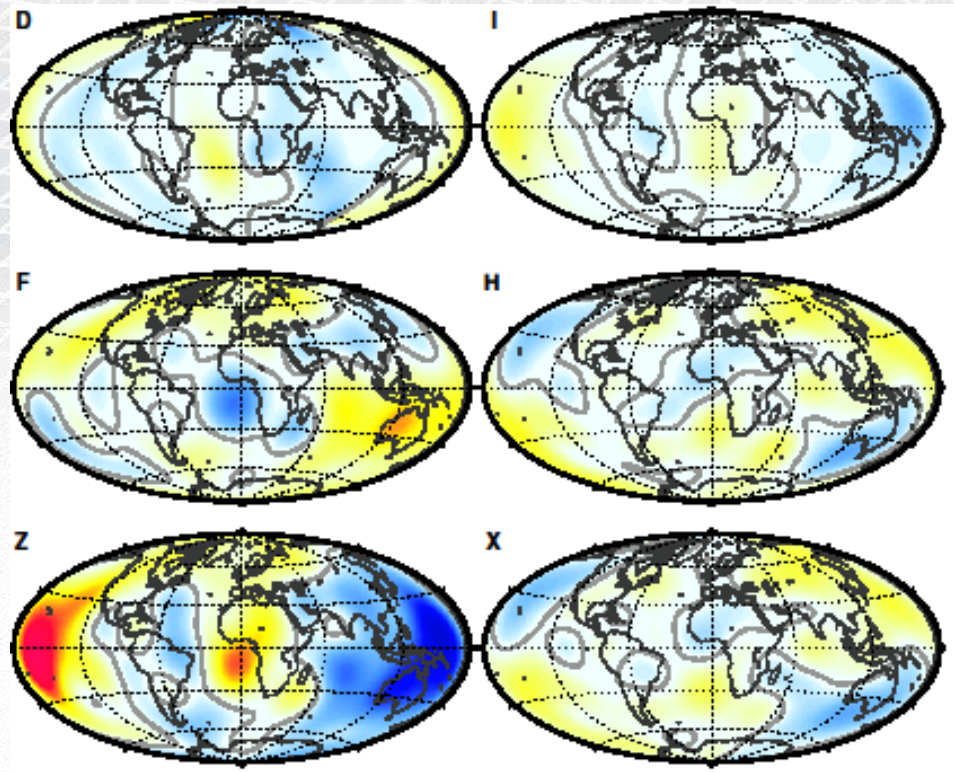

Data to February 2020

BGS model @ 2020.0 - IGRF-13 SV
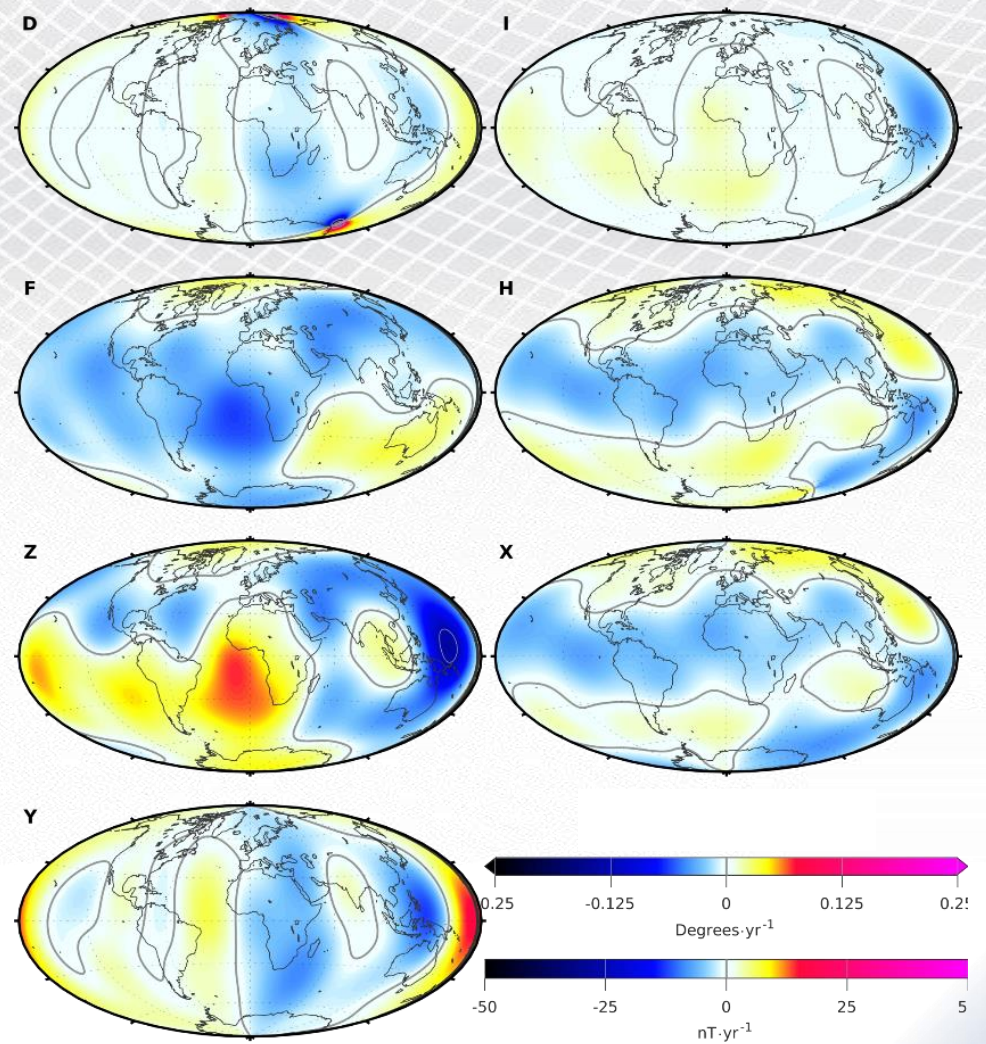


\section{Summary}

- WMM2020 and IGRF-13 are now available, describing the field and rate of change between 2020 and 2025

- BGS provides updated resources and online calculators for use of both models, and for geomagnetic coordinate system conversions

- Significance of non-linear SV and effectiveness of forecast methods can be gauged from retrospective review of IGRF candidates

- Impact of jerks is not isolated in time, it can lead to accrual of increasing discrepancies between linear models and actual field

- During 2015-2020, secular accelerations tended to lead to underestimation of SV amplitude, increasingly over time

- There is no obvious distinction in performance of empirical and physical forecasts for IGRF-12 SV candidates

- Between IGRF-13 SV candidates, the timeliness of data constraining a model is evident, and is likely as big an influencer of accuracy of SV forecasts as forecast methodology, when a linear SV model is considered 


\section{References}

- Alken, P., E. Thébault, C.D. Beggan, et al., 2020. The $13^{\text {th }}$ generation International Geomagnetic Reference Field, Earth Planets Space.

- Chulliat, A., W. Brown, P. Alken, C. Beggan, M. Nair, G. Cox, A. Woods, S. Macmillan, B. Meyer and M. Paniccia, The US/UK World Magnetic Model for 2020-2025: Technical Report, National Centers for Environmental Information, NOAA, doi:10.25923/ytk1-yx35, 2020

- Thébault, E., Finlay, C.C., Alken, P., Beggan, C.D., Canet, E., Chulliat, A., Langlais, B., Lesur, V., Lowes, F.J., Manoj, C. and Rother, M., 2015. Evaluation of candidate geomagnetic field models for IGRF-12. Earth, Planets and Space, 67(1), p.112.

- And all authors contributing to the 9 IGRF-12 SV candidate models

- Torta, J.M., Pavón-Carrasco, F.J., Marsal, S. and Finlay, C.C., 2015. Evidence for a new geomagnetic jerk in 2014. Geophysical Research Letters, 42(19), pp.7933-7940. 